\title{
Joint Cooperative Relay and Cross Layer HARQ for QoS Provisioning in Wireless Sensor Networks
}

\author{
Kun Hua \\ IEEE Senior Member \\ Electrical and Computer Engineering \\ Department \\ Lawrence Technological University, MI, USA \\ khua@|tu.edu \\ Jianjun Yang \\ Department of Computer Science and \\ Information Systems \\ University of North Georgia, GA, USA \\ jianjun.yang@ung.edu
}

\author{
Yuenong Zhu \\ Electrical and Computer Engineering \\ Department \\ Lawrence Technological University, MI, USA \\ yzhu1@ltu.edu
}

\author{
Yin Wang \\ Math and Computer Science Department \\ Lawrence Technological University, MI, USA \\ ywang12@ltu.edu
}

\begin{abstract}
Wireless Sensor Networks (WSN) has drawn significant attention recently as a key important factor especially in sensitive areas, through which, data can be simply collected and transmitted. However, we lack practical WSN that promises a high-speed, real time and energy efficient communication with a small size cell battery. In this paper, we proposed a novel cross-layer Hybrid Automatic Repeat reQuest (HARQ) architecture with QoS Provisioning for Cooperative Wireless Networking, which can optimize system performance by choosing appropriate frame size, modulation and retransmission methods through sensitive analysis. Spatial diversity gain could also be achieved through cooperative relay stations. Our contribution includes two folders: (1) Proposed a novel cross-layer HARQ framework for cooperative wireless networks (2) Proposed optimal QoS provisioning through Choquet-based cross-layer parameters decision.
\end{abstract}

\section{Keywords}

Wireless Sensor Networks, Cooperative, QoS, Cross Layer

\section{INTRODUCTION}

With the explosively increasing demands on services of WSN, high Quality of Service (QoS) becomes a critically important task for the design of communications systems. In WSNs, signal fading, access confliction and the interference between the different users are severe problems. Through the wireless channel, data received has always been attenuated, distorted and degraded greatly. There are several traditional solutions, such as encoding, retransmission, cooperation, and achieving diversities etc. However, none of them are perfect. For example, it is inefficient to encode each data bit in a short range with limited channel attenuation; retransmission is not suitable for error-prone communications under noisy channels; similarly, the ratio of frame head and payload is also negotiable for various channel environments and transmission distances. Therefore, it is a big challenge to optimize frame size, encoding and retransmission strategies.

As shown in figure 1, through a form of a spatial diversity network, cooperative relays could promise both transmission quality and energy efficiency in WSN. To replace the impractical MIMO techniques, cooperative communications form a virtual antenna array ia multiple relay nodes. Cooperation above the physical layer such as cooperative routing and MAC has been proved to improve the network QoS performance. However, separated design on each layer cannot promise an overall optimized system performance. Although the cross-layer design has being investigated as promising solutions, there are critical challenges remained. Meanwhile, the complexity of cross-layer control could hinder the deployment of the solution. The cross-layer control parameters from multiple layers could have different level impacts (sensitivities) on the network performance such as transmission rate, delay tolerance and energy efficiency. It is important to identify key control parameters to reduce the cross-layer control and optimization overheads. In this paper, we studied existing cooperative networks solution from several aspects, and proposed novel cross-layer HARQ architecture for cooperative WSNs. 


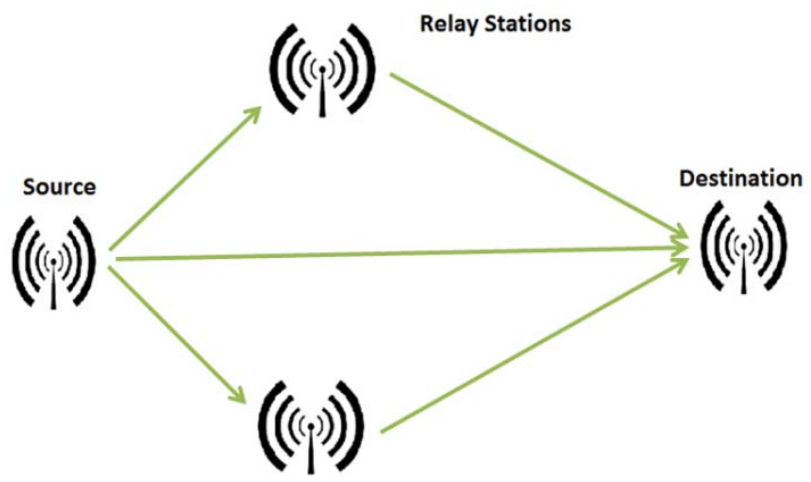

Figure1: Cooperative Wireless Sensor Networks Architecture

The reminder of this paper is organized as follows. Section II reviews relevant literature. The optimal comprehensive resource cost is studied in Section III. The proposed cross layer frame work is presented in Section IV. Simulation results are given in Section V. A Choquet Algorithm solution has been proposed in Section VI and Section VII summarizes our research conclusions.

\section{Literature Review}

The initial idea of cooperative WSN was proposed by Sadek, $\mathrm{Yu}$ and Liu in [1], which introduced an increasing spatial diversity gain through nodes cooperation. In [2], authors analyzed cooperation and relay schemes to maximize the WSN battery lifetime and proposed an energy efficient cooperative communication scheme. In [3], both energy efficiency and transmission quality of various schemes (direct transmission, single-relay cooperation, and multi-relay cooperation respectively) were analyzed. To improve energy efficiency of cooperative relays, paper [4] proposed an idea of packet size optimization. [5] improved the energy efficiency through multiple nodes cooperation and [6] found the optimal frame size for the energy efficient WSN.

Due to the small size and hardware complexity of the mobile terminal, Multiple-input multiple-output (MIMO) might not be practical. To overcome such limitation, Cooperative Diversity (CD) was proposed in [7]. In the suggested CD scheme, several cooperative mobile terminals around the sender will form a virtual cooperative network and transmit data together. Thus, CD offers similar advantages MIMO technique to combat multipath fading [8]. In [9]-[10], Sendonaris has proposed a standard cooperative diversity model which could achieve a capacity improvement. Paper [11] proposed a solution by combining repetition-based and space-time-coded cooperation in a virtual MIMO system, which unfortunately will reduce transmission rates. Hunter lately in [12] proposed a simple but more efficient two users coding cooperation scheme.

In [13], Anghel and Kaveh proposed a medium access control (MAC) algorithm based on IEEE 802.11 which is called Cooperative Diversity MAC. It exploits the cooperative diversity and selects an appropriate cooperative relay to improve the network performance. An efficient spectrum virtual-antenna array cooperation strategy was proposed in [14] to form a multi-cell virtual link with multiple association feature to achieve optimal system performance. In [15], a novel protocol of vehicular cooperative media access control was proposed. The authors theoretically studied the selection of an optimal cooperation relay set using a weighted independent set model. In [16] a novel C-MAC protocol based on IEEE 802.11, was presented. In C-MAC, the receiver combines both source and relay signals, which creates spatial diversity. In [17], the authors introduced distributed information sharing and designed a single-radio cooperative asynchronous multichannel MAC protocol. Paper [18] proposed a Cooperative Diversity MAC (CD-MAC), which applies physical layer CD capability to improve WSN robustness. In [19], the authors gave a brief introduction of cooperative relay network, in which cooperative transmission of primary user is assisted by secondary users. In [20], a new MAC protocol, called CoopMAC, has been proposed to cooperate at the PHY layer with the MAC sub-layer, thereby achieving substantial throughput and improved delay performance. In [21], Markov chain analysis is adopted in Cooperative MAC under IEEE $802.11 \mathrm{n}$ environment. The paper in [22] presented a novel multirate MAC scheme, in which terminals with low data rate will be adaptively helped by higher rate ones.

Paper [24] introduced a cross-layer design of MAC-PHY layer for optimal throughput of wireless networks, which can flexibly choose transmitters and receivers number and modulation types, with various channel states. Optimal throughput will be achieved for multimedia wireless sensor networks by jointly adjusting above mentioned parameters. With static, flat fading, paper [25] studies the tradeoff of diversity and multiplexing for the MARC. Without changes in the devices, it develops MAF and MDF to help the users gain the benefit of cooperative diversity. By using multipath propagation in wireless networks, [26] develops some simple cooperative diversity protocols. [27] presents an analytical framework for HARQ, which aims at the transmission of multimedia with differential encoding. It can be used to define design guidelines of multimedia transmission systems. By adapting to dynamic spectrum environments, paper [8] presents a cognitive space-time-frequency coding method is able to flexibly change its coding schemes. In CR, [28] illustrates the benefits of cooperation, such as reducing the detection time by allowing the cognitive users sharing the same frequency band. Thus it shows a method to increase the overall agility.

\section{Proposed Cross-Layer Design Frame works}

To our investigation, current cooperative communication proposals focus on minimizing the transmission energy cost. Such optimization goal is definitely significant for wireless sensor networks with energy constraints. However, some networks have set high throughput as their optimization goals, according to the multiple access data transmission requests of 4G and 5G systems. Meanwhile some specific multimedia networks also require tight delay and jitter constraints. Since these wireless networking nodes are normally equipped wired power supply without constants of energy, their different optimization goals require different strategies with cooperative transmission. In this paper, we tentatively propose a comprehensive cross-layer link cost with cooperative transmission to achieve a combination of optimization goals on throughput, delay and energy. This link cost is expressed as:

$$
\mathrm{L}_{\mathrm{C}}=\frac{\mathrm{p}_{\mathrm{r}} * \mathrm{D}_{\mathrm{L}}}{\mathrm{R}}
$$

Here, $D_{L}$ refers to the delay of a transmission in a cooperative link; $R$ stands for the bit rate in transmission and

$P_{r}$ represents the required transmission energy at a certain bit rate. Comparing to Rayleigh and other popular distributions in wireless sensor network, the Lognormal distribution can 
provide the best description of the small-scale fading in the WSNs, therefore:

$$
P_{r}=\gamma^{D}+P L(d)+N_{O}
$$

where $\gamma^{D}$ is the received signal to noise ratio (SNR) in $\mathrm{dB}$ at the destination; $N_{O}$ is the power of the Additive White Gaussian Noise (AWGN) at the receiver; and $P L(d)$ is the average path loss between the source and destination WSN nodes with a distance $d$. Here [4],

$$
P L(d)=a+b \log _{10} d+X_{\sigma}
$$

where $a$ and $b$ are reference parameters to best fit WSN path loss environment and $X_{\sigma}$ is the shadowing factor, which is basically a Gaussian distributed random variable featured with zero mean and standard deviation $\sigma$ in $\mathrm{dB}$.

Because the required transmission energy is a function of transmission distance, with the decomposed of each link into $m$ cooperative transmissions, this path cost evolves as:

$$
P C=\sum_{i}^{m} \frac{a * \gamma_{i} *\left(d_{i}\right)^{b} * D_{L, i}}{R_{i}}
$$

where $\gamma_{i}$ is the required SNR to decode the information in the $i$ -th cooperative transmission of a link and $\left(d_{i}\right)^{b}$ is the power attenuation resulted from distance of this transmission with $b$ as the attenuation coefficient taking values from 2 to 4 that depends on various WSN environments. It should be noted that delay $D_{L, i}$ cannot simply take the inverse of the bit rate $R_{i}$ because it involves possible queuing delay and retransmission. Eventually, the cooperative routing goal is to identify a path with the minimum path cost as illustrated in formula (4).

At physical layer, considering the cooperative network shown in Figure 2 where signals are sent from the source $(S)$ to destination $(D)$ over a complex noise channel with path loss $K_{10}^{2}$ to replace previous $\left(d_{i}\right)^{b}$ without losing the generality. In this paper, two relays, $R_{1}$ and $R_{2}$ are used to provide repeated signals through the complex noise environment. Path loss parameters are $\left(K_{12}, K_{13}\right)$ from sender to two relays, and $\left(K_{20}, K_{30}\right)$ from relays to destination, respectively. Here we $n_{R}=n_{D}=N_{O}$. Without losing generality, we also assume $K_{10}$, $K_{12}, K_{13}, K_{20}$ and $K_{30}$ have already been determined at the receiver side [9].

Thereby the instantaneous signal-to-noise ratio (SNR) can be derived by:

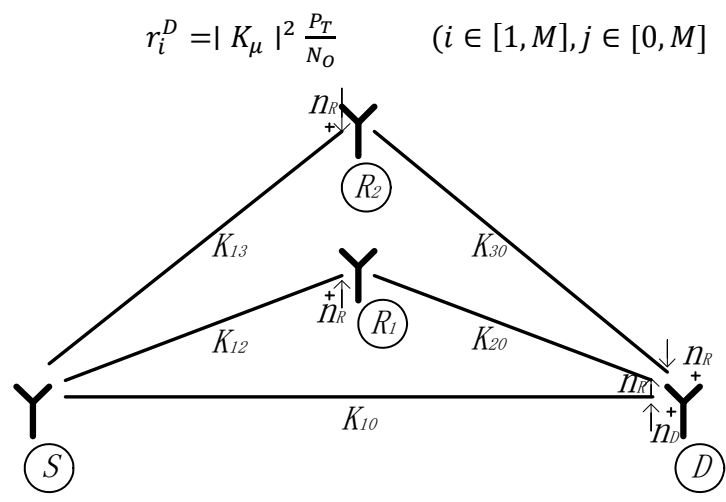

Figure 2: Cooperative network
And from [7], we can achieve as

$$
\gamma=\sum_{k=2}^{M} \frac{1}{\frac{1}{\gamma_{1 k}}+\frac{1}{\gamma_{k 0}}}
$$

The bit error rate for BPSK modulation can be obtained as [7]:

$$
P_{e}=\frac{5}{4 m^{3}} \prod_{k=2}^{3}\left(\frac{1}{\gamma_{1 k}}+\frac{1}{\gamma_{k 0}}\right) \frac{1}{\gamma_{10}}
$$

Thereby, package error rate can be expressed as:

$$
\mathrm{PER}=1-\left(1-\mathrm{P}_{e}\right)^{L}
$$

Where $L$ is the frame size and $L=H+S$, here $H$ and $S$ are header and payload size, respectively.

In this paper, we apply convolutional code with constraint length $(K)$ equals to 3 , code rate $(R)$ equals to $1 / 3$, and the corresponding free distance (dfree) equals to 7 . Thereby in octal representation, the code generator is represented as $(5,7,3)$ [13]. We assign the convolutional code sequence as follows: $d_{1} \oplus d_{2}, d_{1} \oplus d_{2} \oplus d_{3}$ and $d_{1} \oplus d_{2}$ where $\oplus$ denotes the exclusive OR operation.

In aforementioned cooperative scheme, all code words will be sent to the destination through both direct path and relay paths simultaneously as shown in Fig. 2. At the CCC receiver, the block to decode $d_{3}$ is : $\left\{d_{1} \oplus d_{2}, d_{1} \oplus d_{2} \oplus d_{3}\right\}$, and considering those fading relay coefficients introduced in Figure 1 , the received signals will be represented by:

$$
\begin{gathered}
y_{1}=K_{10}\left(d_{1} \oplus d_{2}\right)+n_{1} \\
y_{2}=K_{12} A_{2} K_{20}\left(d_{1} \oplus d_{3}\right)+n_{2} \\
y_{3}=K_{13} A_{3} K_{30}\left(d_{1} \oplus d_{2} \oplus d_{3}\right)+n_{3}
\end{gathered}
$$

where $n_{1}, n_{2}, n_{3}$ are statistically independent and Gaussian distributed as $N\left(0, \sigma_{0}^{2}\right)$.

We choose the amplification factor $A_{i}(i=2,3)$ to match the original transmitted power,

$$
A_{i}=\sqrt{\frac{P_{X}}{p_{x} g_{i}^{2}+N_{O}}}=\sqrt{\frac{\frac{E_{C}}{N_{O}}}{\frac{E_{C}}{N_{O}} K_{1 I}^{2}+1}} \quad(i=2 \text { or } 3)
$$

And so that the Gaussian noise in each relay $n_{R_{i}}(i=2,3)$ can be expressed as:

$$
n_{R_{i}}=A_{i} n_{1}+n_{i}=\sqrt{\frac{\frac{E_{C}}{N_{O}}}{\frac{E_{C}}{N_{O}} K_{1 I}^{2}+1}} * n_{1}+n_{i} \quad(i=2 \text { or } 3)
$$

$n_{R_{i}}$ is Gaussian distributed as $N\left[\frac{\frac{E_{C}}{N_{O}}\left(K_{1 l}^{2}+1\right)+1}{\frac{E_{C}}{N_{O}} K_{1 l}^{2}+1}\right]$ and we take this as $N\left(0, \sigma_{0}\right)$ for expression convenience. Under high $S N R\left(\frac{E_{0}}{N_{0}}\right)$, $\sigma_{R} \approx \sigma_{0}$.

\section{PROPOSED CROSS-LAYER HARQ DESING FOR COOPERATIVE WIRELESS}


The PER of proposed cooperative communication can be represented as:

$$
\mathrm{PER}_{\mathrm{C}}=\sum_{\mathrm{i}=1}^{\mathrm{N}} \mathrm{PER}_{\mathrm{SD}}\left[\mathrm{PER}_{\mathrm{SR}, \mathrm{i}}+\left(1-\mathrm{PER}_{\mathrm{SR}, \mathrm{i}}\right) \mathrm{PER}_{\mathrm{RD}, \mathrm{i}}\right]
$$

where $\mathrm{PER}_{\mathrm{SD}}, \mathrm{PER}_{\mathrm{SR}, \mathrm{i}}$, and $\mathrm{PER}_{\mathrm{RD}, \mathrm{i}}$, are representing packet error rates from source to destination, along the i-th sourcerelay links and from relays to the destination respectively. PER performances of cooperative communications have been shown in figure 7 of section VI.

According to (8), (12) and [31], the expected system data rate:

$$
\begin{gathered}
\overline{R_{b}}=\frac{1}{M} \sum_{i=1}^{N}\left\{R_{b, i}\left[\left(1-P E R_{c}\right)+P E R_{c} \operatorname{PER}_{S R, i}\right]+\right. \\
\left.\frac{1}{\sum_{i=1}^{N} \frac{1}{R_{i}}} P E R_{c}\left(1-\operatorname{PER}_{S R, i}\right)\right\}
\end{gathered}
$$

where the first term stands for a packet transmitted from sender to relays, and received by the destination either successfully, or erroneously. The second term stands for the events that relays retransmit the corrupted packet.

At physical layer, the constraint length and code rate of the Convolutional codes are two major factors to impact QoS provisioning. The proposed scheme will allow the receiver to exploit both time diversity and spatial diversity simultaneously in relay systems [23].

MAC layer parameters include the data rate $S$, contention window size $W, M A C$-frame size $L$, and retransmission limit $T$, Customer number $C_{n}$, etc. For example, under a severe channel condition, the BER will be reduced by decreasing the transmission rate $R$, similarly, the frame error probability will be reduced by decreasing the frame size $L$, and seems the throughput could be improved. But on the other hand, the throughput will be decreased with a lower transmission rate $S$ and extra overhead cost for a smaller frame size $L$ and contention window size $W$. Therefore there are tradeoffs between delay bounds and throughput [7]. Retransmission time $T$ is relative to the performance of delay and Customer number $C_{n}$ is proportional to the energy cost. The network throughput is obtained under different $R, W, L, T$ and $C_{n}$. Obviously, to achieve desired throughput, delay bounds and energy efficiency, an international study need to be done for both parameters $\left\{R, W, L, T, C_{n}\right\}$ at MAC layer and $\{C, R, M, N\}$ at physical layer.

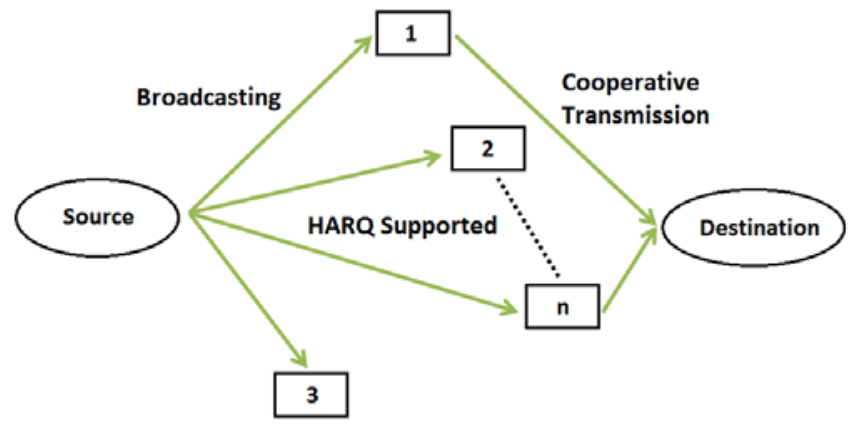

Figure 3: A Cross-layer HARQ architecture for Cooperative Wireless Sensor Networks
HARQ mechanisms are adopted for better reliability. But the maximum retransmission times are upper-bounded for HARQ. If $\mathrm{CRC}$ checking returns correct results at the destination node, ACK packet is transmitted back to relays and further forwarded to the source by relays. Further packet transmission will then be scheduled. In contrast, if CRC checking does not pass, NACK packet will be sent back. But the NACK packet is only forwarded towards selected candidate relay nodes. The selection will be performed by the destination based on certain criterion, such as the signal-to-noise ratio (SNR) threshold based criterion. Then, the selected relay nodes will perform $H A R Q$. The destination node will then combine all received signals from initial transmission and retransmissions. The redecode the signals and check CRC again. If CRC check does not pass, the destination will feed back NACK and require retransmission again. This process is repeated until the maximum number of retransmission is reached. Once successful, an ACK packet will be sent back to relays and relays will also transmit ACK to the source, indicating the successful transmission. This setup is motivated by the fact the channel often varies slowly as compared to the signal bandwidth.

\section{Cross-Layer Design through Choquet Algorithm}

In wireless environment, channel noise and interferences are burst, time varying and location dependent. It is difficult to model the real time wireless network due to the time varying and uncertainty of cross layer control parameters. Unfortunately, all traditional cross-layer approaches usually are exhaustively looking for all available strategies. Due to the computing complexity and control problems, it is normally difficult to achieve the optimal solutions. Especially in cross layered systems, a couple of parameters are commonly contributing their performances, while however, it is a huge challenge to explore their relations mathematically.

Traditional linear measurement might be inappropriate under such complex environment. We propose a nonlinear statistical fuzzy measure model based on non-additive integrals for the key control parameters and their interactions without establishing an exact mathematical model [29]. The distinguishing feature of such model is that the interaction of general parameters in the system can be addressed through nonlinear Choquet integral [30]. The Choquet-based learning algorithm for key parameters selection is described as following. In this algorithm, the significance and interaction among these cross-layer parameters are evaluated.

Choquet-based Learning for Significant Parameters

The Choquet-based learning progress is described in Table 1, in which, interaction among these cross-layer parameters and significance levels have been evaluated.

Table 1: Choquet-based learning process for cross-layer key parameter selection

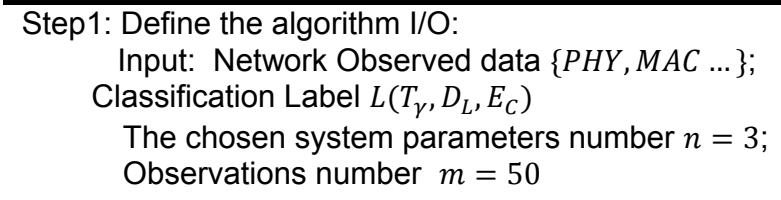

Output: Cross-layer parameters significance value 
$\left\{\mu_{1}, \ldots, \mu_{2^{n-1}}\right\}$, significant cross-layer parameters

Step 2: Input the network training data, coding and decoding the unknown parameters.

Initialize the unknown factors $\mu_{1}, \mu_{2}, \ldots, \mu_{11}, \mu_{12}, \mu_{13}, \ldots$ and $T_{\gamma}, D_{L}, E_{C}$ are coded as binary genes $g_{1}, g_{2}, \ldots$, $g_{2^{n}+2 n}$ respectively. Thus, each gene is a bit string. The length of the bit string depends on the required precision for the solution. Once the genes are generated in some step in the genetic algorithm, they are decoded by formulas $\mu_{i}=2\left(g_{i}-0.5\right)$ in which $i=1,2, \ldots, 2^{n}-1, \quad B=2\left(g_{r}-0.5\right), \quad a=$ $2\left(g_{i}-0.5\right)$ for $i=2^{n}+1, \ldots, 2^{n}+n, b=2\left(g_{i}-0.5\right)$ for $i=2^{n}+n+1, \ldots, 2^{n}+2 n$.

Step 3: Population and chromosomes.

Each chromosome denotes a possible interaction among cross-layer parameters, which can be represented as a gene string, $\left(g_{1}, g_{2}, \ldots, g_{2^{n}+2 n}\right)$

Step 4: The function of evaluating the performance of cross-layer --Chromosomes' fitness.

The fitness function is determined by the target of the optimization, which can be measured by any of the elements or a combination from $T_{\gamma}, D_{L}, E_{C}$. In this algorithm for each chromosome $\left(g_{1}, g_{2}, \ldots, g_{2^{n}+2 n}\right)$, after decoding the genes, we obtain the values of $\mu_{1}, \mu_{2}, \ldots$ and $T_{\gamma}, D_{L}, E_{C}$. Then, based on the given learning data, the corresponding penalized total signed Choquet distance $D_{c}$ from the sample points in the data set to the hyperplane $\mathrm{H}$ can be calculated by (3). Others are the same as $A(c)$

Step 5: Parent selection and producing new chromosomes.

Denoting the fitness of the k-th chromosome in the current population by $F(k)$, based on the probability $p_{k}=F(k) / \sum_{k=1}^{p} F(k)$ to the k-th chromosome, $k=$ $1,2, \ldots, p$. According to the probability distribution $\left\{p_{k} \mid k=1,2, \ldots, p\right\}$ randomly select two chromosomes from the population pool as their parents. Produce two additional chromosome by selecting a given genetic operation, either mutation, or crossover. Repeat $\frac{p}{2}$ times of such procedure to achieve totally $p$ new chromosomes.

Step 6: Renew population.

Calculate the total signed distance of each new chromosome and add these $\mathrm{p}$ chromosomes to the current population. According to the total signed distance of these $2 p$ chromosomes, delete the $p$ worst from them, and then form a new generation of the population.

Step 6: Stop the controller and determine significant parameters based on the important index.

Output the corresponding values of parameters $\mu_{1}, \mu_{2}, \ldots, \mu_{11}, \mu_{12}, \mu_{13}, \ldots$ and $T_{\gamma}, D_{L}, E_{C} ;$ choose important index.

\section{Numerical Results}

We apply the poposed Choquet model in this section for interdependency measure and significance analysis on both Physical and MAC layer parameter set of cross layer WSN. The parameter set includes the number of cooperative nodes, retransmission limits, Physical layer frame size, data rate, and MAC-frame size, etc. Some system parameters are shown in Table 2. The network throughput is obtained by using different combinations of MAC, data rate, retransmissions and frame size.
Table 2: MAC and PHY parameters of the cross layer WSN

\begin{tabular}{c|c}
\hline parameters & values \\
\hline Overhead & 72 bits \\
Data rate & $0.5,1,2,5.5,11 \mathrm{Mbps}$ \\
Modulation Types & $2,4,8,16$ and 64 \\
Transmission power & $-12 \mathrm{dBm}$ to $12 \mathrm{dBm}$ \\
Noise Power & $-100 \mathrm{dBm}$ \\
Channel Model & $\mathrm{d}: 5 \mathrm{~cm}-10 \mathrm{~cm} ; \mathrm{a}: 35 \mathrm{~dB}-50 \mathrm{~dB} ; \mathrm{b}:$ \\
MAC header length & $3-4.5$ \\
ACK length & 112 bits \\
Minimum frame length & 64 bits \\
Maximum frame length & 100 Bytes \\
Retransmission times & 500 Bytes \\
Maximum number of & $1-5$ \\
nodes & 12 \\
Node Placement & Random \\
Minimum number of & 3 \\
nodes &
\end{tabular}

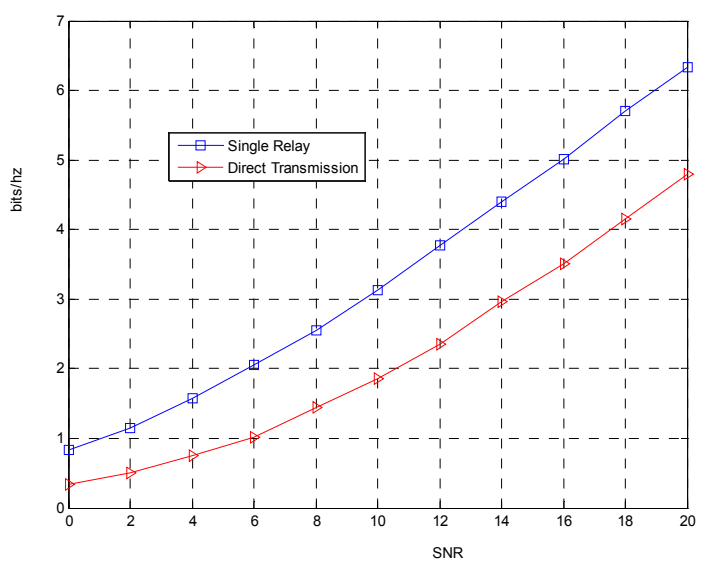

Figure 4: Single Relay cooperation in WSN shows better spectral efficiency performance than Direct Transmission (noncooperation)

In Figure 4, Single Relay cooperation in WSN shows better spectral efficiency performance than Direct Transmission (noncooperation); Figure 5-6 displayed the path cost calculated in (4) and following equations and compared different parameters, including date rate, delay time, retransmission time, transmission power and attenuation coefficient. Figure 7 shows the PER performance for multiple hops $N=2,6,10$ respectively when relays amount $M=3$. Table 3 explains the quantitative significance and interdependency of parameters over the throughput performance. There are four different network conditions considering channel quality (good channel with $\mathrm{BER}=10-6$ and bad channel with $\mathrm{BER}=10-3)$. Each $\mu[i]$ in the table gives the quantitative significance among aforementioned parameters from modulation, data rate, and frame size and retransmissions. 


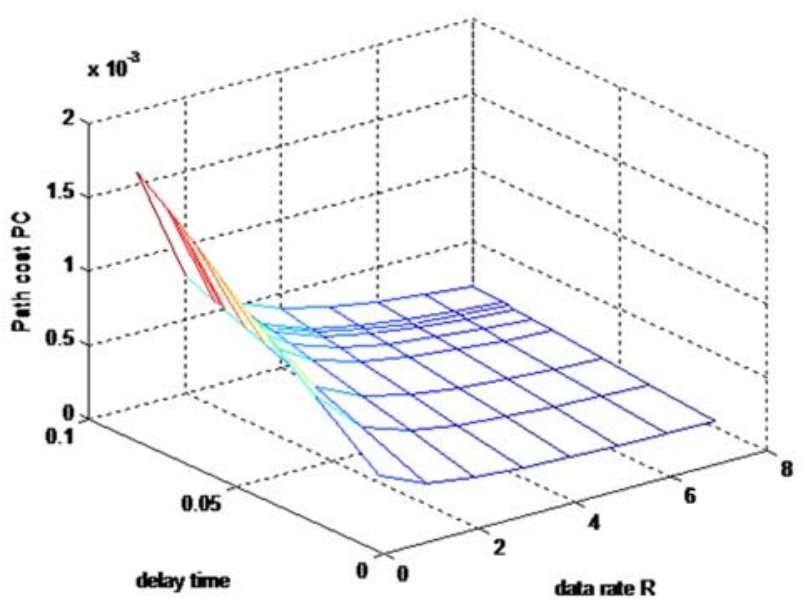

Figure 5: Path cost comparison with delay time (ms) and date rate (Mbps)

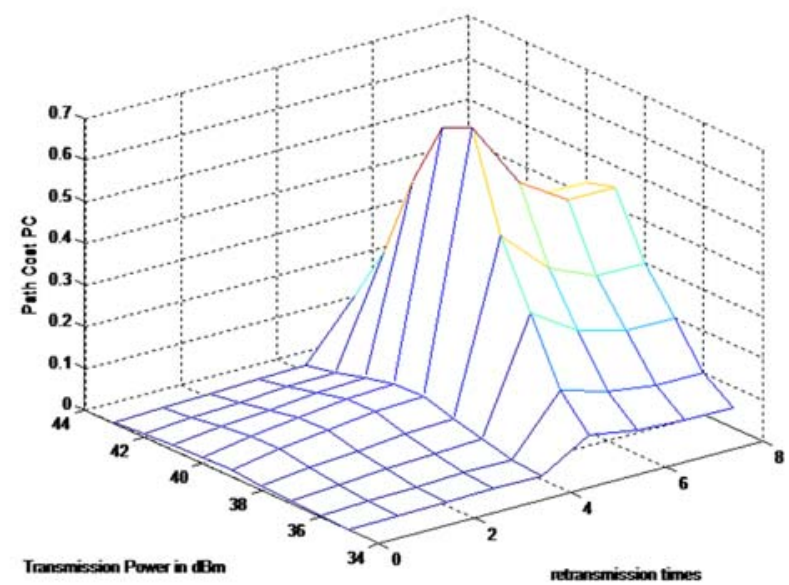

Figure 6: Path cost comparison with retransmission time and transmission power $(\mathrm{dBm})$

Table 3: Experimental data sets

\begin{tabular}{c|c|c|c|c}
$\begin{array}{c}\text { Modulation } \\
\text { Parameter }\end{array}$ & $\begin{array}{c}\text { Noise } \\
\text { Level }\end{array}$ & $\begin{array}{c}\text { Retrans } \\
\text { mission }\end{array}$ & $\begin{array}{c}\text { Frame } \\
\text { Size }\end{array}$ & $\begin{array}{c}\text { Throughput } \\
{[\mathrm{T} / \mathrm{s}]} \\
(\mathrm{bps})\end{array}$ \\
\hline
\end{tabular}

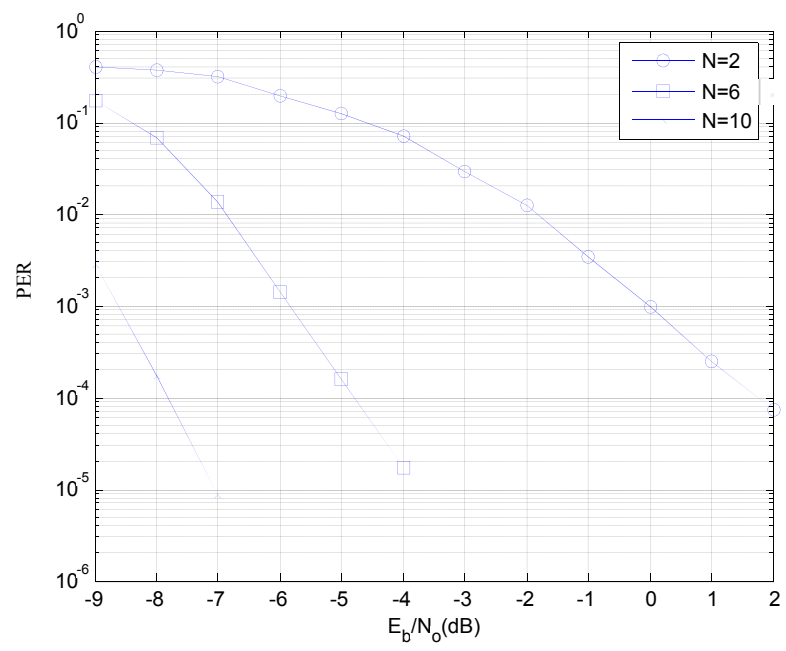

Figure 7: PER performance for different cooperative nodes

\begin{tabular}{c|c|c|c|c}
\hline 2 & 400 & 1 & 64 & 4 \\
2 & 800 & 2 & 81 & 8 \\
2 & 1200 & 4 & 100 & 18 \\
$\ldots$ & $\ldots$ & $\ldots$ & $\ldots$ & $\ldots$ \\
4 & 400 & 1 & 64 & 21 \\
4 & 800 & 2 & 81 & 28 \\
4 & 1200 & 4 & 100 & 41 \\
$\ldots$ & $\ldots$ & $\ldots$ & $\ldots$ & $\ldots$ \\
8 & 400 & 1 & 64 & 47 \\
8 & 800 & 2 & 81 & 49 \\
8 & 1200 & 4 & 100 & 56 \\
$\ldots$ & $\ldots$ & $\ldots$ & $\ldots$ & $\ldots$ \\
16 & 400 & 1 & 64 & 92 \\
16 & 800 & 2 & 81 & 98 \\
16 & 1200 & 4 & 100 & 113 \\
$\ldots$ & $\ldots$ & $\ldots$ & $\ldots$ & $\ldots$ \\
64 & 400 & 1 & 64 & 173 \\
64 & 800 & 2 & 81 & 194 \\
64 & 1200 & 4 & 100 & 205 \\
& & & & \\
\hline
\end{tabular}

\section{Conclusion}

In this paper, we proposed a Cross-layer HARQ design framework for cooperative wireless body area networks, which can efficiently decide the cooperative operation and choose appropriate cross-layer parameters through sensitive analysis. We applied Choquet learning-based decision method for cooperation choice and cross-layer parameter selection, including retransmission time, modulation, frame size and attenuation coefficient on both physical and MAC layers.

\section{References}

[1] A. K. Sadek, W. Yu, and K. J. R. Liu, "On the energy efficiency of cooperative communications in wireless sensor networks," ACM Trans.Sensor Networks, vol. 6, no. 1, Dec. 2009.

[2] Z. Zhou, S. Zhou, S. Cui, and J. Cui, "Energy-efficient cooperative ommunication in a clustered wireless sensor network," IEEE Trans. Vech.Technol., vol. 57, no. 6, pp. 3618-3628, Nov. 2008.

[3] Y. Chen, J. Teo, J. C. Y. Lai, E. Gunawan, K. S. Low, C. B. Soh, and P. B. Rapajic, "Cooperative communications in ultra-wideband wireless body area networks: channel 
modeling and system diversity analysis," IEEE J. Sel. Areas Commun., vol. 27, no. 1, pp. 5-16, Jan. 2009.

[4] B. Braem, B. Latre, I. Moerman, C. Blondia, and P. Demeester, "The need for cooperation and relaying in short-range high path loss sensor networks," in Proc. 2007 Int. Conf. Sensor Technol. Appl. (SENSORCOMM 2007), pp. 566-571.

[5] X. Huang, H. Shan, X. Shen .On Energy Efficiency of Cooperative Communications in Wireless Body Area Networks , IEEE ;2011.

[6] K.S. Deepak, A.V. Babu, "Packet Size Optimization for Energy Efficient Cooperative Wireless Body Area Networks", India Conference (INDICON), 2012.

[7] A. Ribeiro, C. X. Cai and G. B. Giannakis, "Symbol error probabilities for general cooperative links," IEEE Trans on Wireless Commun., vol. 4, no.3, pp. 1264 - 1273, May 2005.

[8] S. Nagaraj and M. Bell, "A coded modulation technique for cooperative diversity in wireless networks," Proceedings of IEEE International Conference on Acoustics, Speech, and Signal Processing (ICASSP '05), vol. 3, pp.525-528, Mar 2005.

[9] A. Sendonaris, E. Erkip and B. Aazhang, "User cooperation diversity-part I: system description," IEEE Trans on Commun., vol. 51, no. 11, pp. 1927-1938, Nov 2003.

[10] A. Sendonaris, E. Erkip and B. Aazhang "User cooperation diversity part II: Implementation aspects and performance analysis," IEEE Trans on Commun., vol. 51, no. 11, pp. 1939-1948, Nov 2003.

[11] N. J. Laneman and G. W. Wornell, "Distributed spacetimecoded protocols for exploiting cooperative diversity in wireless networks," IEEE Trans. Inform. Theory, vol. 49, no. 10, pp. 2415-2425, Oct 2003.

[12] T. E. Hunter and A. Noosratinia, "Cooperative diversity through coding," Proc. IEEE Int. Symp.

[13] P. A. Anghel and M. Kaveh, "Exact symbol error probability of a cooperative network in a Rayleigh-fading environment," IEEE Trans on Wireless Communications, vol.3, no. 5 Sep. 2004.

[14] Sangman Moh; Chansu Yu; Seung-Min Park; Heung-Nam Kim; Jiwon Park; , "CD-MAC: Cooperative Diversity MAC for Robust Communication in Wireless Ad Hoc Networks," Communications, 2007. ICC '07. IEEE International Conference on , vol., no., pp.3636-3641, 24-28 June 2007

[15] Yao Hua; Qian Zhang; Zhisheng Niu; , "A cooperative MAC protocol with virtual-antenna array support in a multiAP WLAN system," Wireless Communications, IEEE Transactions on , vol.8, no.9, pp.4806-4814, September 2009

[16] Jin Zhang; Qian Zhang; Weijia Jia; , "VC-MAC: A Cooperative MAC Protocol in Vehicular Networks," Vehicular Technology, IEEE Transactions on , vol.58, no.3, pp.1561-1571, March 2009

[17] Huan Jin; Xinbing Wang; Hui Yu; Youyun Xu; Yunfeng Guan; Xinbo Gao; , "C-MAC:A MAC Protocol Supporting Cooperation in Wireless LANs," Wireless Communications and Networking Conference, 2009. WCNC 2009. IEEE , vol., no., pp.1-6, 5-8 April 2009

[18] Luo, T.; Motani, M.; Srinivasan, V.; , "Cooperative Asynchronous Multichannel MAC: Design, Analysis, and Implementation," Mobile Computing, IEEE Transactions on , vol.8, no.3, pp.338-352, March 2009

[19] Moh, S; Yu, C; , "A Cooperative Diversity-based Robust MAC Protocol in Wireless Ad Hoc Networks," Parallel and
Distributed Systems, IEEE Transactions on , vol.PP, no.99, pp.1-1, 0

[20] Qian Zhang; Juncheng Jia; Jin Zhang; , "Cooperative relay to improve diversity in cognitive radio networks," Communications Magazine, IEEE , vol.47, no.2, pp.111117, February 2009

[21] Verde, F.; Korakis, T.; Erkip, E.; Scaglione, A.; , "A Simple Recruitment Scheme of Multiple Nodes for Cooperative MAC," Communications, IEEE Transactions on , vol.58, no.9, pp.2667-2682, September 2010

[22] Wong, D.T.C.; Shajan, M.R.; Ying-Chang Liang; Chin, F.P.S.; , "Performance Analysis of Cooperative MAC in IEEE 802.11n for Multiclass Traffic," Vehicular Technology Conference, 2007. VTC-2007 Fall. 2007 IEEE 66th , vol., no., pp.205-209, Sept. 30 2007-Oct. 32007

[23] Tao Guo; Carrasco, R.; , "CRBAR: Cooperative relaybased auto rate MAC for multirate wireless networks," Wireless Communications, IEEE Transactions on , vol.8, no.12, pp.5938-5947, December 2009

[24] Kun, H., Zhu, Y., Wang, W., \& Wang, H. (2014, October). Cross-Layer Design of MAC-PHY for Optimal Capacity of Multimedia Wireless Sensor Networks. Paper presented at Research in Adaptive and Convergent Systems, Towson, MD.

[25] Chen, D. \& Laneman, J.N. (2006, March). The DiversityMultiplexing Tradeoff for the Multiaccess Relay Channel. Paper presented at 40th Annual Conference on Information Sciences and Systems, Princeton, NJ.

[26] Laneman, J.N., Tse, D.N.C. \& Wornell, Geogory W. (2004). Cooperative Diversity in Wireless Networks: Efficient Protocols and Outage Behavior. IEEE Transactions on Information Theory, Volume: 50, Issue: 12, $3062-3080$.

[27] Badia, L., Levorato, M. \& Zorzi, M. (2009, June). Analysis of Selective Retransmission Techniques for Differentially Encoded Data. Paper presented at IEEE International Conference on Communications, Dresden.

[28] Ganesan, G. \& Li, Y. (2007). Cooperative Spectrum Sensing in Cognitive Radio, Part I: Two User Networks. IEEE Transactions on Wireless Communications, Volume: 6, Issue: $6,2204-2213$.

[29] Fang, H., Rizzo, M. L., Wang, H., Espy, K. A., "A new nonlinear classifier with a penalized signed fuzzy measure using effective genetic algorithm", Pattern Recognition (Elsevier), 2010.

[30] Z. Wang. A new model of nonlinear multiregressions by projection pursuit based on generalized Choquet integrals [J]. IEEE International Conference on Fuzzy Systems, 2002: 1240-1244

[31] S. Wang, N. Zhou, H. Zhao and W. Du, "A cross-layer adaptive incremental cooperative relaying scheme for wireless sensor networks", Information and Communications Technologies (ICT 2014), pp1-6, 2014 